\title{
Cannabis-derived cannabidiol and nanoselenium improve gut barrier function and affect bacterial enzyme activity in chickens subjected to $C$. perfringens challenge
}

Paweł Konieczka ${ }^{12^{*}} \mathbb{D}$, Dominika Szkopek ${ }^{1}$, Misza Kinsner ${ }^{1}$, Bartosz Fotschki ${ }^{3}$, Jerzy Juśkiewicz ${ }^{3}$ and Joanna Banach ${ }^{4}$

\begin{abstract}
Revealing the multifocal mechanisms affecting cross-talk between Clostridium perfringens pathogenesis and the host response is an urgent need in the poultry industry. Herein, the activity of Cannabis sativa-derived cannabidiol (CBD) and selenium nanoparticles (Nano-Se) in modulating the host response to Clostridium perfringens challenge was investigated in broiler chickens subjected to a mild infection model. The infected chickens exhibited no clinical manifestations, confirming the potential hazard of pathogen transmission to the food chain in the commercial sector. However, both CBD and Nano-Se affected the responses of chickens to C. perfringens challenge. The beneficial actions of both agents were manifested in the upregulated expression of genes determining gut barrier function. Both CBD and Nano-Se promoted shifts in gut bacterial enzyme activity to increased energy uptake in challenged chickens and upregulated potential collagenase activity. There was no opposite effect of CBD and Nano-Se in mediating the host response to challenge, whereas an additive effect was evidenced on the upregulation of gene determining gut integrity. Collectively, these findings indicate that understanding the action mechanisms of CBD and Nano-Se is of great interest for developing a preventive strategy for $C$. perfringens infection in broilers.
\end{abstract}

Keywords: cannabidiol, selenium nanoparticles, necrotic enteritis, gut health, microbiota activity, broiler chicken

\section{Introduction}

Poultry production is steadily increasing worldwide. In addition to certain welfare issues associated with intensive rearing systems, there are new challenges that not only are potentially hazardous for birds but also could lead to contamination of final products for human consumption. Necrotic enteritis (NE) caused by the anaerobic bacterium Clostridium perfringens (or C. perfringens) is responsible for major economic loss to the poultry

\footnotetext{
*Correspondence: p.konieczka@ifzz.pl

${ }^{1}$ Department of Animal Nutrition, The Kielanowski Institute of Animal

Physiology and Nutrition, Polish Academy of Sciences, Instytucka 3, 05-110 Jabłonna, Poland

Full list of author information is available at the end of the article
}

industry due to compromised bird performance and increased rates of morbidity and mortality [1]. This issue has attracted great attention since the preventive use of antibiotics in farm animals has been banned [2]. Thus, there is an urgent need in the poultry sector to develop an effective strategy based on dietary intervention that can limit or prevent the occurrence of NE. Numerous strategies have been investigated to prevent or reduce the negative impact of $C$. perfringens in birds, but the obtained data are inconclusive, mainly because the pathogenic mechanisms of this pathogen are poorly understood [3-6]. Two main factors are responsible for the inconsistent outcomes among studies: (i) the effects of NE-predisposing factors, including coccidia coinfection, 
nutrition, and bird age [7], and (ii) the basing of conclusions on results obtained/extrapolated from clinical cases with differences in NE disease severity (i.e., subclinical vs. severe). For example, the magnitude of NE lesions affects the expression of genes associated with mucin synthesis in gut tissue [8]. This was evidenced in a study by Forder et al. [9], who found a depressed mRNA level of MUC2 and MUC13 gene expression in the gut tissue as a result of a greater severity of NE in broilers. The authors speculated that it might be associated with an increased chance of further infection. However, considering the current implications for the poultry sector, a focus on understanding the mechanism underlying mild NE is very important, particularly in broiler chickens, because broilers are raised under commercial conditions for an extremely short duration (6-7 weeks). Thus, potential interventions to help broilers recover from advanced $\mathrm{NE}$ during the final growth period are rather scarce. In contrast, birds with mild NE typically do not exhibit any symptoms or disorders; thus, these birds might serve as a vector for the delivery of $C$. perfringens and/or its toxins to the food chain $[10,11]$.

The major site of Clostridium perfringens-host interactions in chickens is the gastrointestinal tract (GIT). Thus, this environment is an important anatomical region in which to investigate mechanisms involved in the response to Clostridium perfringens challenge and for the development of agents that may potentially regulate this response [12]. Identifying the mechanisms by which NE can be limited may provide new insights into biosecurity. Bioactive components with potential modulatory activity towards GIT health include cannabinoids (CBD) from cannabis plants (Cannabis sativa), which have recently attracted increasing attention [13]. Research on the therapeutic potential of CBD has led to many important discoveries in medicine, including the discovery of the action of CBD in regulating food intake, nausea, emesis, gastric secretion, gastroprotection, GIT motility, ion transport, visceral sensation, intestinal inflammation and cell proliferation in the gut $[13,14]$. Thus, potential regulation by $\mathrm{CBD}$ may be an effective intervention in the management of NE at the initial stage. Although the anti-inflammatory activity of CBD has been extensively studied in human medicine, its influence on GIT function, including immune competence, in veterinary practice is unknown because of the difficulty in identifying the precise dose of $\mathrm{CBD}$ for animal treatment. Another agent that exhibits biological activity in the GIT is selenium (Se). Accumulating evidence has shown that the Se status in birds directly affects the immune system, including aspects such as disease resistance, survival, growth performance, fertility, and metabolic processes that determine meat quality [15-18]. However, a recent report indicated that Se has indirect regulatory actions on the GIT through modulation of a host protective response to NE challenge and can alter the residual microbiota, contributing to improved intestinal physiology, allowing increased nutrient absorption and enhancing the immune response to NE [19]. Because nanosized selenium particles (Nano-Se) have been reported to have higher bioavailability but lower toxicity than Se in other forms [20], they could be used to effectively modulate GIT functionality while maintaining compliance with nutritional safety recommendations. Therefore, in this study, we applied-for the first time in a chicken modeldietary treatment with CBD extracted from hemp and with Nano-Se to investigate the activity of these agents in modulating the gut response to Clostridium perfringens infection. Additionally, we adopted a challenge model that was effective in the development of mild lesions in broilers. This model was meant to be helpful for investigating changes in bird responses occurring in nonadvanced NE and will likely have potential applications in NE-preventive studies and implications for the poultry industry.

\section{Materials and methods Chemical composition of cannabis extract and Nano-Se} Hemp panicles (Cannabis sativa) obtained from plants were harvested in 2019 at the Institute of Natural Fibres \& Medicinal Plants, Poznań, Poland. Plant material was collected, cut and dried at room temperature. The supercritical carbon dioxide extract of hemp was obtained from the Supercritical Extraction Plant, Institute of New Chemical Synthesis, Puławy, Poland. Parameters of extraction: pressure; 250 bar, temperature; $60{ }^{\circ} \mathrm{C}$, and flow rate of $40 \mathrm{~kg} \mathrm{CO}_{2} / 1 \mathrm{~kg}$ of spent hemp. Hemp extract contained $12 \% \mathrm{CBD}, 0.49 \%$ tetrahydrocannabinol and $0.38 \%$ tetrahydrocannabinolic acid (Institute of Natural Fibers and Medicinal Plants, Poznań, Poland). Nano-Se was used in the form of a nanopowder with an average particle size of $10-45 \mathrm{~nm}$, specific surface area (SSA) of approximately $30-50 \mathrm{~m}^{2} / \mathrm{g}$, and purity of $99.9 \%$, according to the manufacturer's declaration (American Elements, CA, USA).

\section{Bacterial strain and culture conditions}

C. perfringens type $\mathrm{G}$ strain 56 was isolated from cases of infection in Belgium. The strain was previously confirmed to be $\alpha$-toxin- and NetB toxin-positive and $\beta$-toxin- and enterotoxin-negative according to supplier declaration (Ghent University, Merelbeke, Belgium). The strain was grown anaerobically in autoclaved brain heart infusion broth (Sigma-Aldrich) containing beef heart $(5 \mathrm{~g} / \mathrm{L})$, calf brain $(12.5 \mathrm{~g} / \mathrm{L})$, disodium hydrogen phosphate $(2.5 \mathrm{~g} / \mathrm{L}), \mathrm{D}(+)$-glucose $(2 \mathrm{~g} / \mathrm{L})$, peptone $(10 \mathrm{~g} / \mathrm{L})$, 
and sodium chloride $(5 \mathrm{~g} / \mathrm{L})$ at $37{ }^{\circ} \mathrm{C}$ overnight in a jar containing an atmosphere of $10 \% \mathrm{CO}_{2}, 10 \% \mathrm{H}_{2}$, and $80 \%$ $\mathrm{N}_{2}$.

\section{Chicken experiment and diets}

A total of 360 one-day-old male broilers of the Ross 308 strain purchased from a local commercial hatchery were randomly distributed among the five dietary treatment groups with 8 replicate cages of nine chickens per group. Chickens were fed a standard starter diet for Ross 308 broilers for 8 days. The conditions of the room were maintained according to standard management practices for commercial chicken houses. Beginning on day 9 , chicks were transitioned to a grower diet, which was formulated to meet or exceed the requirements for broiler chickens [21]. The basal grower diet based on wheat (50.76\%), soybean meal $(21.76 \%)$, triticale (15.54\%), fish meal (5.18\%), and rapeseed meal $(4.15 \%)$ made the gut environment favourable for $C$. perfringens growth. The chickens were fed a control grower diet (unchallenged negative CON group and challenged positive CON group), CON supplemented (on top) with $15 \mathrm{~g} / \mathrm{kg}$ Cannabis sativa extract (CBD treatment group), a diet supplemented with NanoSe instead of inorganic Se (Nano-Se treatment group), or a diet supplemented with both additives (CBD + Nano-Se treatment group). All grower diets were cold-pelleted in our laboratory in a CL-2 CPM (CA, USA) laboratory pellet mill.

\section{Establishment of the experimental NE model}

Infection of chickens was performed according to a previously described protocol with some modifications [22]. At 15, 16, 17 and 18 days of age, chickens were infected with $1 \mathrm{~mL}$ (per os directly into the crop) of overnight culture Clostridium perfringens inoculate (as described above), prepared fresh for each day of challenge, whereas chickens in the nonchallenged group received the same dose of sterilized broth medium. Before $C$. perfringens challenge, $1 \mathrm{~mL}$ of a coccidial cocktail containing the following Eimeria $(E)$ species was administered to all birds at 14 and 15 days of age to create a favourable gut environment for $C$. perfringens colonization: $E$. acervulina, 5000 oocytes; E. maxima, 3500 oocytes; E. mitis, 5000 oocytes; E. praecox, 5000 oocytes; E. tenella, 5000 oocytes (Laboratorios HIPRA S.A., Spain).

\section{Evaluation of chicken performance}

The body weight (BW) and feed intake (Fint) of the chickens were recorded, and performance indices, including the FCR, were calculated for various periods (challenge period, postchallenge period, and all experimental periods) to investigate the specific response of the chickens to the dietary treatments throughout the experimental duration.

\section{Determination of the collagen level and collagenase concentration in gut tissue}

The collagen level in gut tissue was measured as the hydroxyproline content according to the protocol described by Gawronska-Kozak [23]. In brief, samples of gut tissue (middle jejunum) were collected from 8 chickens in each dietary treatment group at 23 and 35 days of age $(n=8)$. Mucosa samples were homogenized with a glass homogenizer on ice in $2 \mathrm{~mL}$ of cold $\left(4{ }^{\circ} \mathrm{C}\right)$ phosphate-buffered saline (Sigma-Aldrich by Merck) and stored overnight at $4{ }^{\circ} \mathrm{C}$. To analyse the level of total collagen, a total of $0.20-0.25 \mathrm{~g}$ of mucosa was used. Subsequently, $0.5 \mathrm{~mL}$ aliquots were hydrolysed with $0.25 \mathrm{~mL}$ of $6 \mathrm{~N} \mathrm{HCl}$ for $4.5 \mathrm{~h}$ at $120^{\circ} \mathrm{C}$. Hydroxyproline (SigmaAldrich by Merck) concentrations from 0 to $20 \mu \mathrm{g} /$ $\mathrm{mL}$ were used to construct a standard curve. Twenty microlitres of each sample and the standard dilutions were added to a well plate and incubated for $20 \mathrm{~min}$ at room temperature with $50 \mu \mathrm{L}$ of chloramine $\mathrm{T}$ solution (282 $\mathrm{mg}$ of chloramine T, $2 \mathrm{~mL}$ of n-propanol, $2 \mathrm{~mL}$ of distilled water and $16 \mathrm{~mL}$ of citrate acetate buffer [5\% citric acid, $7.24 \%$ sodium acetate, $3.4 \%$ sodium hydroxide and $1.2 \%$ glacial acetic acid]). Next, $50 \mu \mathrm{L}$ of Ehrlich's solution (2.5 g of 4-[dimethyloamino] benzaldehyde, $9.3 \mathrm{~mL}$ of n-propanol and $3.9 \mathrm{~mL}$ of $70 \%$ perchloric acid) was added, and the plate was incubated for $15 \mathrm{~min}$ at $65{ }^{\circ} \mathrm{C}$. Samples were then cooled, and the plate was read in a microplate reader (Multiskan Sky Microplate Spectrophotometer, Thermo Fisher Scientific) at $550 \mathrm{~nm}$.

Collagenase concentration was measured in the same tissue samples with the corresponding enzyme-linked immunosorbent assay (ELISA) kits (LS BioScience, Seattle, WA, USA) according to the manufacturer's protocol. In brief, gut samples were collected and rinsed in PBS to remove digesta. Thereafter, the samples were homogenized in $10 \mathrm{~mL}$ of PBS with a glass homogenizer on ice and subsequently lysed by ultrasonication. The homogenate was centrifuged at $5000 \times g$ for 5 min and was then stored at $-80{ }^{\circ} \mathrm{C}$ for further analysis. The standard curve was generated using known antigen concentrations at a wavelength of $450 \mathrm{~nm}$, and the enzyme concentrations in the samples were determined by comparison to the standard curve. Each sample was measured in duplicate, and a standard curve was plotted separately for each ELISA plate.

\section{RNA extraction and real-time quantitative PCR}

Gut samples collected from 23-day-old chickens were additionally analysed by RT-PCR to measure the mRNA 
expression levels of GLP2, HSP70, TLR4, JAM2, ZO-1, and TFF2. RNA was isolated from tissue according to the protocol described by Konieczka et al. [24] A GeneMATRIX Universal RNA Purification Kit (EURX Ltd., Gdańsk, Poland) was used to isolate total RNA.

The yield of isolated RNA was estimated spectrophotometrically (Nanodrop, NanoDrop Technologies, Wilmington, DE, USA), and the integrity was assessed electrophoretically by separation on an agarose gel. Subsequently, RNA isolated from gut tissue $(800 \mathrm{ng} / \mathrm{mL})$ was reverse transcribed to complementary DNA (cDNA) using an NG dART RT Kit (EURX Ltd., Gdańsk, Poland) according to the manufacturer's protocols. Primers specific for each gene were designed using Primer 3 software (Whitehead Institute, Cambridge, MA, USA) and synthesized by Genomed (Warsaw, Poland) (Table 1). Real-time qPCR was performed in a Bio-Rad CFX 96 thermocycler (Bio-Rad Laboratories, Inc., CA, USA) according to the following programme: (i) enzyme activation (one cycle at $95{ }^{\circ} \mathrm{C}$ for $15 \mathrm{~min}$ ), (ii) denaturation ( 35 cycles at $95^{\circ} \mathrm{C}$ for $10 \mathrm{~s}$ ), (iii) annealing ( 35 cycles at $55-58{ }^{\circ} \mathrm{C}$ for $10 \mathrm{~s}$ ) and (iv) elongation (35 cycles at $72{ }^{\circ} \mathrm{C}$ for $20 \mathrm{~s}$ ). Melt curve analysis was performed from $65-95^{\circ} \mathrm{C}$ at $0.1{ }^{\circ} \mathrm{C} / \mathrm{s}$ intervals. Each reaction included a negative control (without the cDNA template). The samples were analysed in duplicate. Gene expression levels were normalized with the comparative quantitation option of the Rotor qPCR Bio-Rad CFX 96 (Bio-Rad Laboratories, Inc., CA,
USA), and the values were calculated using the supplied Expression Software Tool. The glyceraldehyde-3-phosphate dehydrogenase (GAPDH) and $\beta$-actin (ACTB) genes were used as endogenous controls for normalization of gene expression.

\section{Measurement of extracellular and intracellular activity of glycolytic enzymes}

The enzymatic activity of the caecal microbiota as a response to dietary treatments was evaluated based on the extra- and intra-cellular activity of bacterial enzymes. The caecal activity of the enzymes $(\alpha-$ and $\beta$-glucosidase, $\alpha$ - and $\beta$-galactosidase, $\beta$-glucuronidase, $\alpha$-arabinopyranosidase, and $\beta$-xylosidase) was determined based on the amounts of $p$-nitrophenol (PNP) or $o$-nitrophenol (ONP) liberated from the respective nitrophenylglucoside substrates, as described [25] and adopted previously [26]. Enzyme activities were measured in caecal digesta collected from chickens at 23 and 35 days of age. In brief, the substrate solution $(0.3 \mathrm{~mL}$; $5 \mathrm{mM})$ and caecal digesta dilution $(0.2 \mathrm{~mL} ; 1: 10 \mathrm{v} / \mathrm{v}$; centrifuged at $7211 \times g$ for $15 \mathrm{~min}$ ) were prepared in $100 \mathrm{mM}$ phosphate buffer ( $\mathrm{pH}$ 7.0) and were then mixed and incubated at $39^{\circ} \mathrm{C}$. The activity of enzymes (micromoles per hour per gram of digesta) released from the cells into the caecal environment was measured by quantifying PNP or ONP at wavelengths of 400 and $420 \mathrm{~nm}$, respectively. The reaction was terminated with $0.25 \mathrm{M}$ cold sodium carbonate $(1 \mathrm{~mL})$. The procedure described above was

Table 1 Genes and primers used in the study.

\begin{tabular}{|c|c|c|c|c|c|}
\hline Gene & Primer & Sequence $\left(5^{\prime}-3^{\prime}\right)$ & $\begin{array}{l}\text { Melting temperature } \\
\left({ }^{\circ} \mathrm{C}\right)\end{array}$ & Product size (nt) & GenBank accession no. \\
\hline \multirow[t]{2}{*}{ ACTB } & Forward & CGGACTGTTACCAACACCCA & 58 & 115 & NM_205518 \\
\hline & Reverse & TCCTGAGTCAAGCGCCAAAA & & & \\
\hline \multirow[t]{2}{*}{ GAPDH } & Forward & GCACGCCATCACTATCTT & 58 & 82 & NM_204305 \\
\hline & Reverse & GGACTCCACAACATACTCAG & & & \\
\hline \multirow[t]{2}{*}{ ZO-1 } & Forward & TCGCTGGTGGCAATGATGTT & 58 & 89 & XM_413773 \\
\hline & Reverse & TTGGTCTCCTTCCTCTAATCCTTCTT & & & \\
\hline \multirow[t]{2}{*}{ GLP2 } & Forward & TGTGTTCAGACGGTAAGG & 58 & 127 & NM_001163248 \\
\hline & Reverse & TCATCCAGTGCCATCTTC & & & \\
\hline \multirow[t]{2}{*}{ HSP70 } & Forward & GGCAATAAGCGAGCAGTG & 58 & 146 & NM_001006685 \\
\hline & Reverse & CGAGTGATGGAGGTGTAGAA & & & \\
\hline \multirow[t]{2}{*}{ TFF2 } & Forward & ACTACCCTACTGAGAGAACAAA & 58 & 143 & XM_416743 \\
\hline & Reverse & CTGAAGAACCTGCTCAACTG & & & \\
\hline \multirow[t]{2}{*}{ TLR4 } & Forward & CAAGCACCAGATAGCAACA & 58 & 146 & FJ915527 \\
\hline & Reverse & CACTACACTACTGACAGAACAC & & & \\
\hline \multirow[t]{2}{*}{ JAM2 } & Forward & TCСТСССАСТAСТССAATATG & 58 & 134 & XM_026849998 \\
\hline & Reverse & ACTGCCTGTTCCTGTCTT & & & \\
\hline
\end{tabular}

ACTB $\beta$-actin, GAPDH glyceraldehyde-3-phosphate dehydrogenase, ZO-1 Zonula occludens-1, GLP2 Glucagon-like peptide-2, HSP70 Heat Shock Protein 70, TFF2 Trefoil Factor 2, TLR4 Toll-like Receptor 4, JAM2 Junctional Adhesion Molecule 2. 
used to measure extracellular activity. Additionally, the total activity (extracellular + intracellular) of the respective enzymes was measured. For this measurement, a similarly diluted caecal sample containing microbial cells was disrupted with glass beads and a FastPrep-24 homogenizer (MP Biomedicals, Santa Ana, CA). The rest of the procedure was identical to that used to measure extracellular enzymatic activity. Intracellular activity was calculated by subtracting the extracellular activity from the total activity. Standard curves for PNP and ONP were used in the respective calculation formulas. Extracellular enzymatic activity was also expressed as a percentage of total enzymatic activity.

\section{Statistical analysis}

Data are presented as the means $(n=8$ chickens per group), and variability is expressed as the pooled standard error of the mean (SEM) values. Differences between groups were assessed using one-way ANOVA with the least significant difference (LSD) test. The significance level was set at $\mathrm{P}<0.05$. Correlations between tissue collagen content and collagenase concentration were assessed with Pearson correlation analysis. Statistical calculations were performed in STATGRAPHICS Centurion XVI ver. 16.1.03 software.

\section{Results}

\section{Response of chickens to $C$. perfringens infection}

The observation of the gut structures showed no development of advanced infection (data not shown). Different regions of the GIT, including the duodenum, jejunum, and ileum, exhibited no signs of necrosis, which in advanced stadium might be manifested as confluent mucosal necrosis of large parts of the gut segments, a collapsed intestinal lumen, a lack of turgor, a thin and friable intestinal wall, advanced necrosis of the gut mucosa, and apparent multifocal haemorrhage in different areas depending on advancements [3, 27]. Thus, the infection model applied herein was suitable for the investigation of the host response to Clostridium perfringens infection of a low severity.

\section{Performance response to treatments}

We studied the response of chickens to challenge and to dietary treatments in different stages of growth to gain deeper insight into the response of chickens during and after challenge. The performance response of the chickens to the dietary treatments calculated for different experimental feeding periods is shown in Figure 1. The body weight gain (BWG) in the day 9-23 and day 24-35 feeding periods did not differ between the experimental groups $(\mathrm{P}>0.05)$, whereas the $\mathrm{BWG}$ calculated for the overall experimental diet feeding period was significantly lower in the challenged positive $\mathrm{CON}$ group and the CBD group than in the unchallenged negative CON group $(\mathrm{P}<0.01)$. The Fint was similar among all treatment groups for all feeding periods $(\mathrm{P}>0.05)$. However, the feed conversion ratio (FCR) was compromised in all experimental groups compared to the unchallenged negative CON group in the day 9-23 feeding period $(\mathrm{P}<0.01)$ but was not affected in the other feeding periods $(\mathrm{P}>0.05)$.

\section{Regulatory effect of CBD and Nano-Se on collagen degradation and collagenase concentration in gut tissue} Challenge with $C$. perfringens resulted in a significantly lower collagen content in gut tissue than that observed in unchallenged chickens (Figure 2). This phenomenon was observed in chickens at 23 days and 35 days of age $(\mathrm{P}<0.001)$; moreover, the dietary treatments did not counteract this effect in chickens of either age (panel A). Regarding the collagenase concentration in gut tissue (panel B), in 23-day-old chickens, no significant differences were observed among the treatments, whereas in the older chickens, feeding with Nano-Se significantly upregulated collagenase concentration compared to that in the unchallenged negative $\mathrm{CON}$ group, and feeding with $\mathrm{CBD}+\mathrm{Nano}-\mathrm{Se}$ upregulated collagenase concentration compared to that in both the unchallenged negative CON group and CON-positive groups $(\mathrm{P}=0.004)$. No correlation was observed between collagen content and collagenase concentration in 23-day-old chickens $(\mathrm{R}=0.236, \mathrm{P}=0.142)$, whereas a weak negative correlation between collagen content and collagenase concentration was observed in the gut of older chickens $(\mathrm{R}=-0.344, \mathrm{P}=0.030)$.

\section{CBD- and Nano-Se-mediated changes in the transcript levels of select genes determining gut integrity in challenged birds}

The mRNA expression patterns of selected gut integrityregulating genes in gut tissue are presented in Figure 3. Generally, treatment with Nano-Se and treatment with $\mathrm{CBD}+$ Nano-Se significantly upregulated the mRNA expression of genes such as glucagon-like peptide-2 (GLP2), Toll-like receptor 4 (TLR4), and junctional adhesion molecule 2 (JAM2) (all $\mathrm{P}<0.001)$. The expression of the zonula occludens-1 ( $\mathrm{ZO}-1)$ gene was upregulated in all experimental groups compared to the unchallenged negative $\mathrm{CON}$ group and $\mathrm{CON}$-positive groups $(\mathrm{P}<0.001)$. The expression level of the heat shock protein 70 (HSP70) gene did not differ between the challenged positive CON group and CBD treatment groups but was significantly higher in the remaining treatment groups 


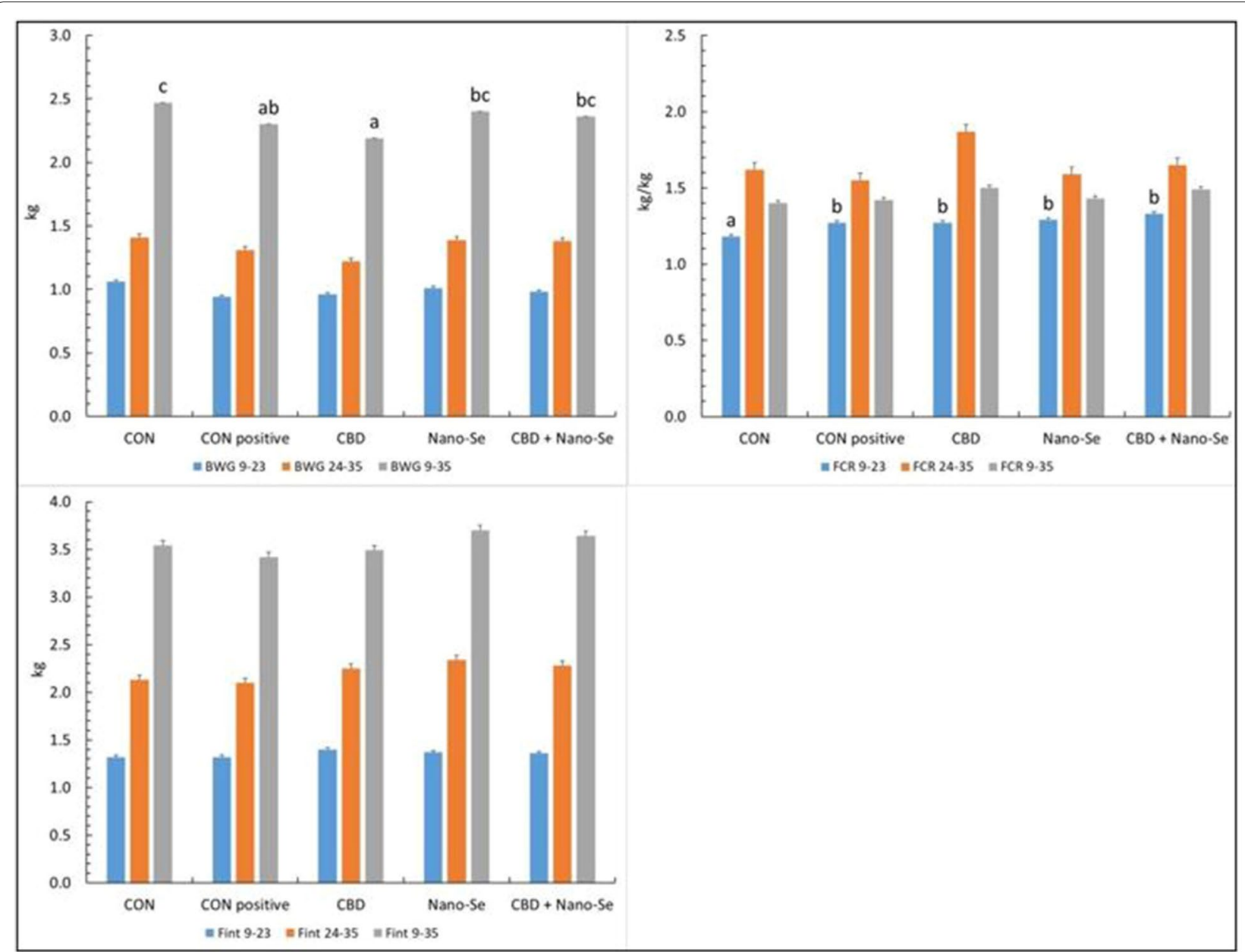

Figure 1 The body weight gain (BWG), feed intake (Fint), and feed conversion ratio (FCR) were calculated for different experimental periods. The chickens were fed a control diet (CON), CON supplemented with $15 \mathrm{~g}$ of hemp extract (CBD)/kg of diet, CON supplemented with $0.5 \mathrm{mg}$ of nanosized selenium particles (Nano-Se)/kg of diet, or CON supplemented with both additives (CBD+Nano-Se). At 15, 16, 17 and 18 days of age, chickens in each experimental group and the positive control group (CON-positive) were orally infected with $1 \mathrm{~mL}$ of $10^{8} \mathrm{CFU} / \mathrm{mL}$. perfringens inoculate. A coccidial cocktail $(1 \mathrm{~mL})$ was administered before $C$. perfringens challenge to all chickens. ${ }^{a}, b, c$ The different letters indicate significant differences $(P<0.01)$. The error bars indicate the mean standard error values for the 8 chickens in each dietary treatment group.

$(\mathrm{P}=0.020)$. The expression level of the trefoil factor 2 (TFF2) gene did not differ among the dietary treatment groups $(\mathrm{P}>0.05)$.

\section{CBD and Nano-Se affected microbiota enzyme activity}

The activity levels of selected bacterial enzymes in the caecal digesta in 23-day-old and 35-day-old chickens are presented in Figures 4 and 5. Both the C. perfringens challenge and the dietary treatments caused shifts in the bacterial enzyme activity. In 23-day-old birds, the intracellular activity of $\beta$-glucuronidase (panel $\mathrm{E}$ ) was higher in the unchallenged negative $C O N$ group $(\mathrm{P}<0.001)$, but the release rate was lower than that of the $\mathrm{CON}$-positive group $(\mathrm{P}=0.039)$. In the same birds, the extracellular activity and release rate of $\alpha$-arabinopyranosidase (panel
G) were higher in the unchallenged negative $\mathrm{CON}$ group than in the $\mathrm{CON}$-positive group $(\mathrm{P}=0.016$ and $\mathrm{P}=0.014$, respectively). The remaining enzyme activities did not differ significantly in challenged vs. not challenged birds $(P>0.05)$. In older birds, the extracellular and total activity of $\alpha$-glucosidase (panel A) were higher in the unchallenged negative $\mathrm{CON}$ group than in the $\mathrm{CON}$-positive group (for both $\mathrm{P}<0.001$ ). The extracellular activity of $\beta$-glucuronidase (panel E) was higher in the $\mathrm{CON}$-positive group than in unchallenged birds $(\mathrm{P}<0.001)$, whereas other enzyme activities did not differ significantly between challenged and unchallenged birds $(P>0.001)$. The dietary treatments caused shifts in the intracellular and extracellular activities of different enzymes. In 23-day-old chickens, the intracellular and total activities 

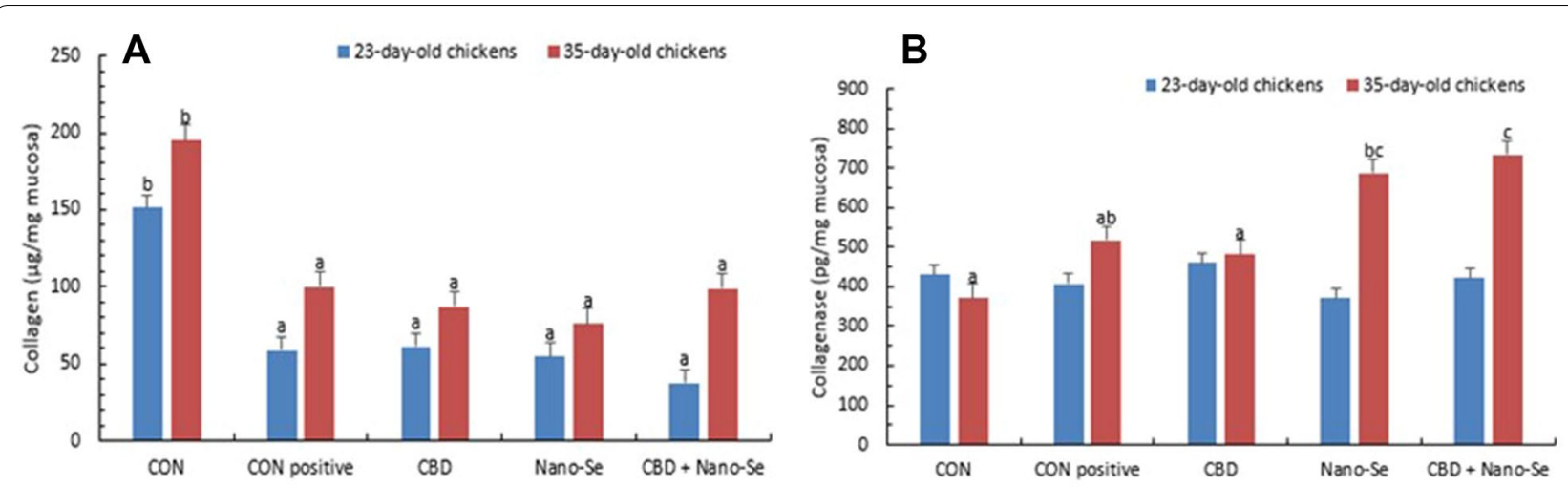

Figure 2 Collagen content and collagenase concentration in the gut tissue of chickens. Chickens were fed a control diet (CON), CON supplemented with $15 \mathrm{~g}$ of hemp extract (CBD)/ kg of diet, CON supplemented with $0.5 \mathrm{mg}$ of nanosized selenium particles (Nano-Se)/ $\mathrm{kg}$ of diet, or CON supplemented with both additives (CBD+ Nano-Se). At 15, 16, 17 and 18 days of age, the chickens in each experimental group and the positive control (CON-positive) group were orally infected with $1 \mathrm{~mL}$ of $10^{8} \mathrm{CFU} / \mathrm{mL}$ C. perfringens inoculate. A coccidial cocktail (1 mL) was administered before $C$. perfringens challenge to all chickens. ${ }^{a},{ }^{b},{ }$ The different letters indicate significant differences $(P<0.01)$. The error bars indicate the mean standard error values for the 8 chickens in each dietary treatment group.

of $\alpha$-glucosidase (panel A) were reduced, but the release rate was increased in chickens fed CBD and/or Nano-Se compared chickens fed the control diets $(\mathrm{P}<0.001)$. The same patterns were observed for $\beta$-glucosidase activity (panel B), but the release rate was significantly higher in the CBD- and/or Nano-Se-supplemented groups than in the CON-positive group $(\mathrm{P}<0.001)$. The extracellular activity of $\beta$-glucuronidase (panel $\mathrm{E}$ ) was the highest in the unchallenged negative $\mathrm{CON}$ group and $\mathrm{CON}$-positive groups $(P=0.004)$, the intracellular activity was highest in the unchallenged negative $\mathrm{CON}$ group $(\mathrm{P}<0.001)$, and the total activity was decreased in all CBD- and/or Nano-Se-supplemented groups $(\mathrm{P}<0.001)$. However, the $\beta$-glucuronidase release rate was significantly lower in the unchallenged negative $\mathrm{CON}$ group than in the other treatment groups $(\mathrm{P}=0.039)$. The intracellular activity of $\beta$-xylosidase (panel F) was significantly lower in all treatment groups than in the unchallenged negative CON group and CON-positive groups $(\mathrm{P}<0.001)$, but the total activity was decreased only in the CBD and NanoSe treatment groups $(\mathrm{P}=0.010)$. The highest release rates were found in all the CBD and/or Nano-Se supplemented groups, but these rates differed significantly between the Nano-Se and CBD + Nano-Se treatment groups and the unchallenged negative $\mathrm{CON}$ group and CON-positive groups $(\mathrm{P}=0.033)$. The extracellular activity of $\alpha$-arabinopyranosidase (panel G) was highest in the unchallenged negative $\mathrm{CON}$ group compared to the other treatment groups $(\mathrm{P}=0.016)$, the intracellular activity was lower in the Nano-Se and CBD+Nano-Se treatment groups than in the challenged positive $\mathrm{CON}$ group $(P=0.013)$, and the total activity was significantly lower in the Nano-Se and CBD + Nano-Se treatments than in the unchallenged negative $\mathrm{CON}$ group $(\mathrm{P}=0.024)$. The $\alpha$-arabinopyranosidase release rate was significantly higher in the unchallenged negative $\mathrm{CON}$ group than in the $\mathrm{CON}$-positive and CBD groups $(\mathrm{P}=0.014)$. The activities of these specific enzymes shifted in 35-dayold chickens (Figure 5) as follows: the extracellular and total activities of $\alpha$-glucosidase (panel A) were reduced in all groups compared to the unchallenged negative CON group $(\mathrm{P}<0.001)$. Among the treatment groups, the extracellular activity of $\alpha$-galactosidase (panel $C$ ) was the lowest, but the intracellular activity was the highest in the CBD+Nano-Se treatment group $(\mathrm{P}<0.001)$. The $\alpha$-galactosidase release rate was the lowest in the $\mathrm{CBD}$ and $\mathrm{CBD}+$ Nano-Se treatment groups $(\mathrm{P}<0.001)$. The extracellular activity of $\beta$-galactosidase (panel $\mathrm{D}$ ) was increased significantly in the CBD treatment group $(P=0.002)$, whereas the intracellular and total activities were increased in the $\mathrm{CBD}$ and $\mathrm{CBD}+\mathrm{Nano-Se}$ treatment groups compared with the remaining treatment groups $(\mathrm{P}<0.001)$. The $\beta$-galactosidase release rate was the lowest in the CBD + Nano-Se treatment group $(\mathrm{P}<0.001)$. In the CBD + Nano-Se treatment group, the extracellular and total activities of $\beta$-glucuronidase (panel E) were reduced compared with those in the unchallenged negative $C O N$ group $(P<0.001$ and $P=0.004$, respectively). $\mathrm{CBD}$ supplementation caused significant increases in the extracellular, intracellular, and total activities of $\alpha$-arabinopyranosidase $(\mathrm{P}<0.001) \quad$ (panel F) and $\beta$-xylosidase $(\mathrm{P}=0.013, \mathrm{P}=0.007$ and $\mathrm{P}<0.001$, respectively) (panel G). 


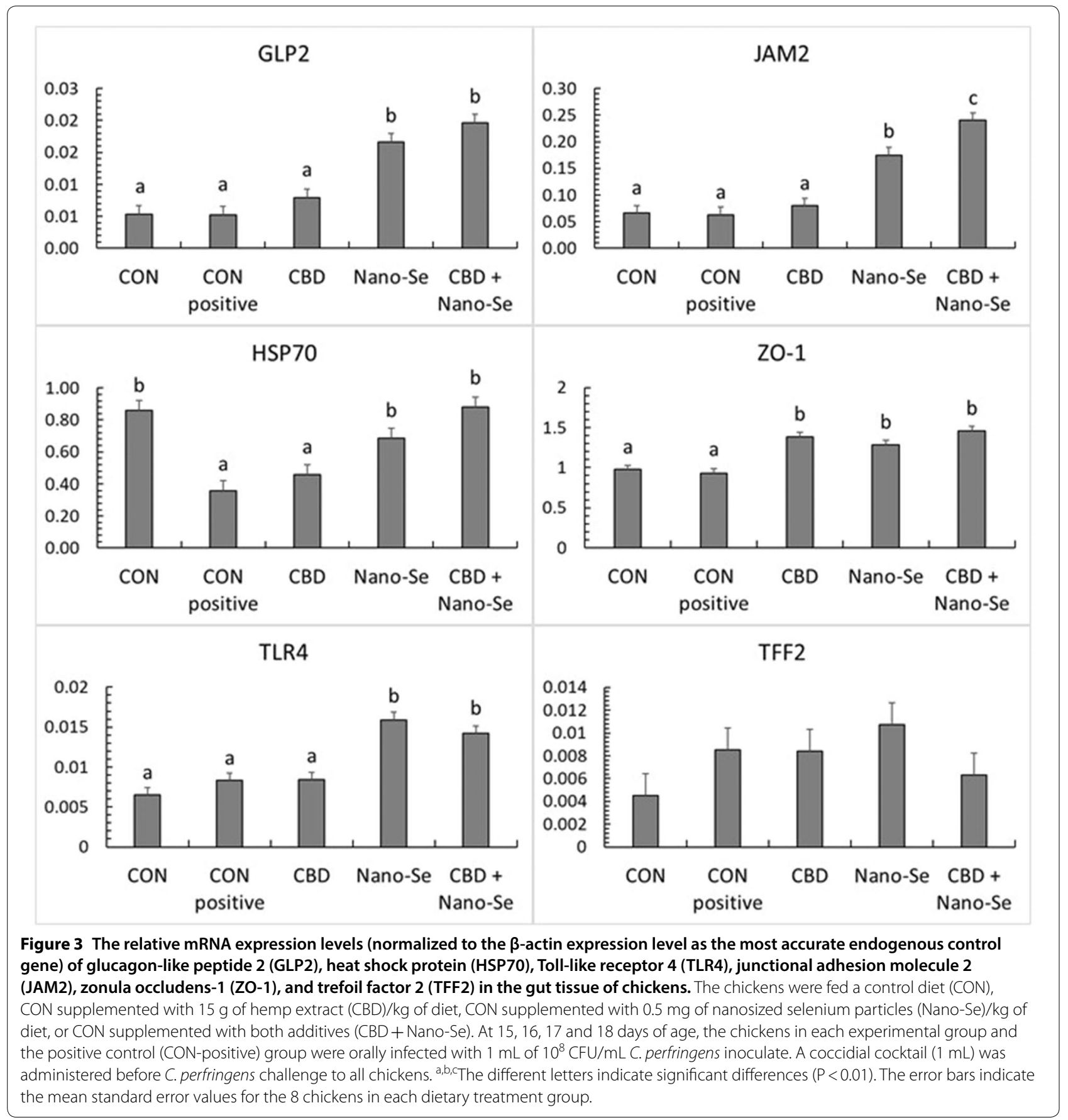

\section{Discussion}

To the best of our knowledge, this is the first study investigating the bioactive properties of CBD and Nano-Se in broilers with potential activity towards gut health and functionality modulation. In particular, the mechanism underlying the action of CBD has not yet been fully elucidated, but it may represent a biological activity in the regulation of inflammation by a close affinity to the processes mediating necrosis and inflammation of the chicken gut. Because NE is an urgent problem in the poultry industry, this bioactive agent may represent an interesting solution in preventive strategies. Therefore, additives that can contribute to the health status of birds while maintaining bird performance indices are currently of high interest for the poultry sector. 


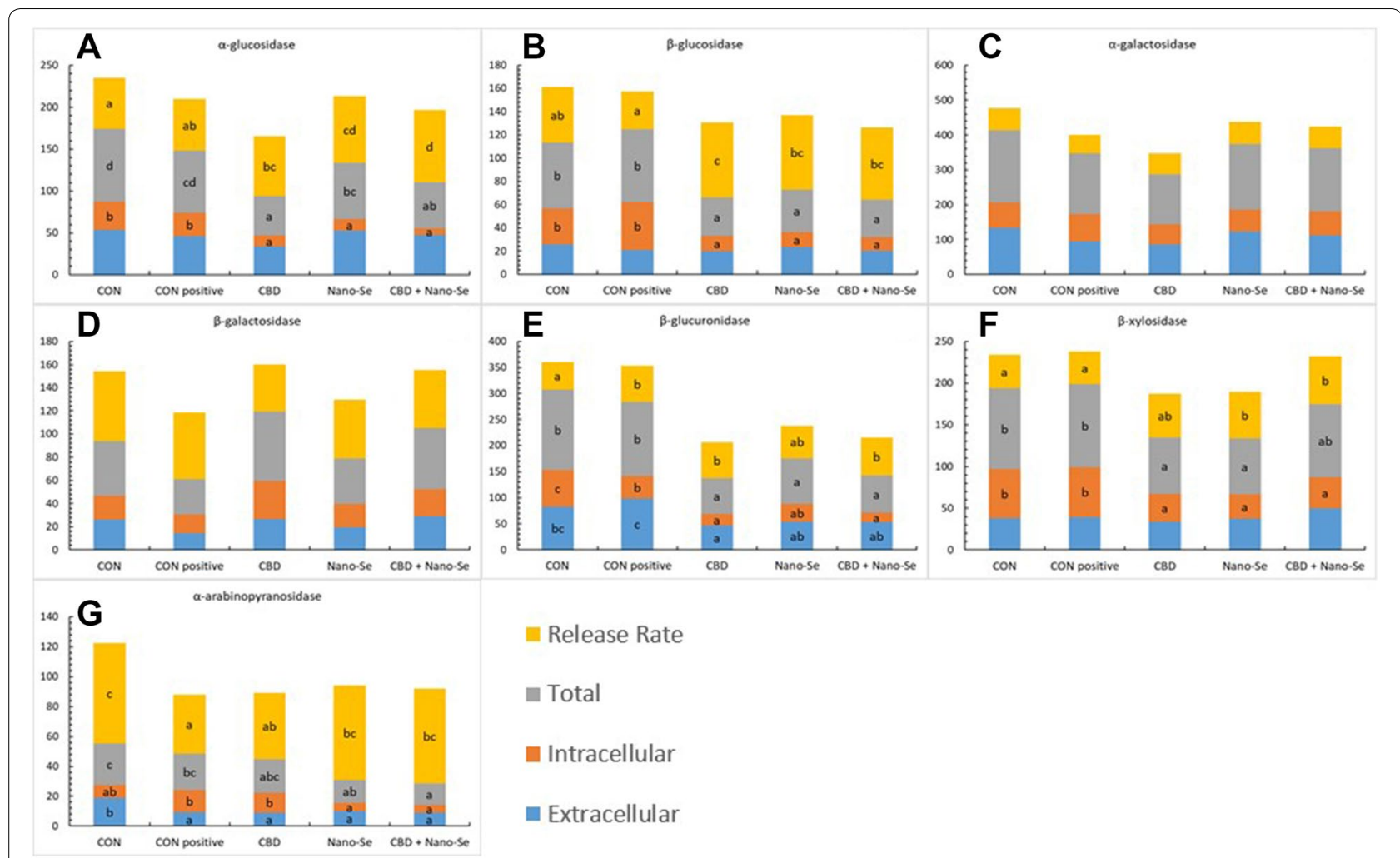

Figure 4 Bacterial enzyme activity [ $\mu \mathrm{mol} /(\mathrm{h}$ g protein)] and release rate (\%) in the caecal digesta of 23-day-old chickens. The chickens were fed a control diet (CON), CON supplemented with $15 \mathrm{~g}$ of hemp extract (CBD)/kg of diet, CON supplemented with 0.5 mg of nanosized selenium particles (Nano-Se)/kg of diet, or CON supplemented with both additives (CBD+Nano-Se). At 15, 16, 17 and 18 days of age, the chickens in each experimental group and the positive control (CON-positive) group were orally infected with $1 \mathrm{~mL}$ of $10^{8} \mathrm{CFU} / \mathrm{mL}$ C. perfringens inoculate. A coccidial cocktail $(1 \mathrm{~mL})$ was administered before $C$. perfringens challenge to all chickens. ${ }^{a}, \mathrm{~b}, \mathrm{c}$ The different letters indicate significant differences $(P<0.01)$. The error bars indicate the mean standard error values for the 8 chickens in each dietary treatment group.

The performance indices of the chickens reported in the present study confirmed that the challenge only marginally affected their growth. According to other authors [28], subclinical NE was associated with depressed performance and mild lesions in the small intestine. Interestingly, however, during the experimental period in which the challenge was performed (days 9-23), neither the BWG nor Fint of the chickens changed significantly, but the FCR was compromised in all challenged chickens compared to the unchallenged chickens. This pattern suggests that the response of the chickens to infection is more complex than a simple reduction/increase in feed consumption and that mechanisms controlling feed utilization are involved. Calefi et al. [29] demonstrated that gut-brain axis interactions during NE challenge may play a key role in the bird response. Our findings confirmed that infection with Clostridium perfringens may occur with no signs of overt growth suppression and is a potential threat to the poultry industry; therefore, this condition warrants special attention.
The occurrence of NE has been linked to the ability of $C$. perfringens to adhere to the host extracellular matrix structures through the adhesive ability to collagen, which is the main structural component of connective tissue and thus potentially the initial pathogenic target [30,31]. Collagen disruption was clearly observed in our study; the gut tissue collagen content was significantly decreased in both 23- and 35-day-old birds challenged with $C$. perfringens. Regarding the dietary treatments applied in the present study, neither CBD nor Nano-Se (or the combination) significantly affected the collagen content in gut tissue. On the other hand, there was no significant effect of $C$. perfringens challenge on the gut total concentration of collagenase enzymes (negative $\mathrm{CON}$ group vs. CONpositive group) in 23- and 35-day-old birds. These results are in contrast to those reported by Van Damme et al. [32], who reported reduced expression of selected host collagenases in necrotic gut tissue as a result of $C$. perfringens challenge. The contradictory findings may indicate that the severity of the infection may play a 


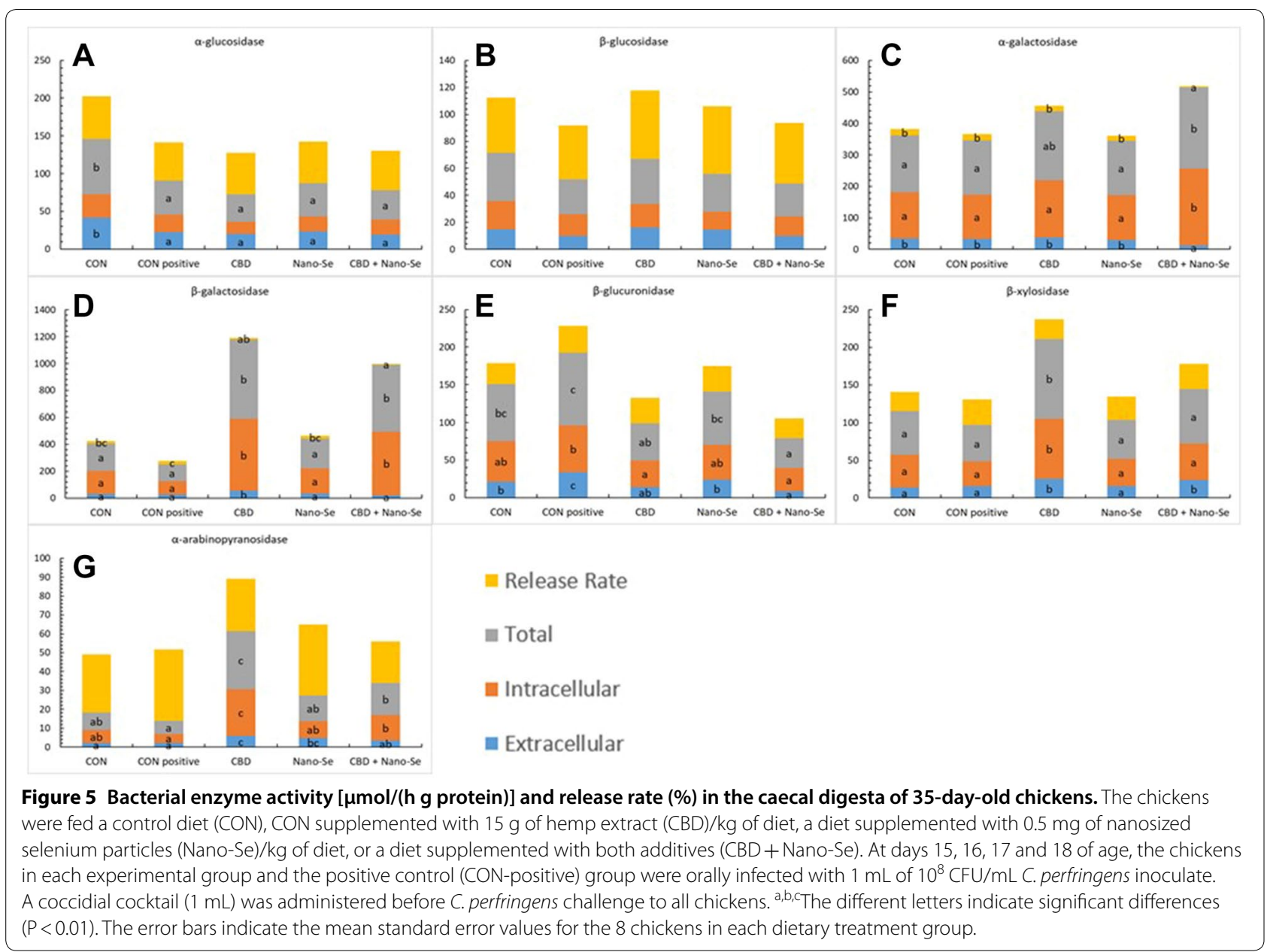

role in the specific response of host collagenase enzyme activity since in our study, no advanced necrosis developed. However, the total concentration of collagenase enzymes in the gut tissue in older chickens was significantly increased in the Nano-Se treatment group compared to the negative CON group and was significantly increased in the CBD + Nano-Se treatment group compared to both the negative CON group and the CONpositive group.

Collagenase enzymes are members of the matrix metalloproteinase (MMP) family of protease enzymes, whose primary function is degrading and remodelling the extracellular matrix in gut tissue [33, 34]. The secretion of collagenolytic enzymes and activation of host MMP cascades, which induce collagen degradation and, consequently, gut enteritis, have been reported to be key $C$. perfringens virulence factors in NE [35]. Our findings indicated that CBD and Nano-Se did not manifest an opposite action in mediating host collagenase enzyme secretion in the gut tissue, whereas Nano-Se promoted this process. This may be considered unfavourable for the host gut. Aguirre et al. [36] reported that supplementation of the chicken diet with resin acids had a protective effect on the intestinal barrier integrity under optimal (with no challenge) conditions, which were linked to decreased activities of collagen-degrading enzymes in the gut tissue. Another report [37] indicated that raisin acid supplementation improved broiler performance in both optimal and NE-challenged conditions, but the authors failed to show a clear mode of action for this effect; thus, the significant role of collagen-degrading enzymes in mediating the host response to $C$. perfringens challenge cannot be excluded.

Based on our results, there was mostly no significant response as a result of $C$. perfringens challenge in mRNA expression of selected genes in the jejunal tissue investigated as relevant indicators of gut integrity in chickens. This is in contrast to other reports (reviewed by Awad et al.) [38], in which it was revealed that C. perfringens infection causes an overall perturbation in the expression of genes determining gut integrity. However, such an effect of $C$. perfringens is mostly reported in birds with 
advanced forms of infection, which may partially explain the different responses reported in the present study. Tight junction proteins (TJPs) are the key molecules maintaining epithelial barrier integrity. TJPs enable the passage of ions inside cells but prevent pathogens and their toxins from entering cells, thus acting as gatekeepers [39]. Although data regarding the specific action of CBD in the bird gut are lacking, studies in different models suggest that CBD possibly modulates the gut neuroimmune axis and mediates gut inflammation via complex cross-talk with other immune cell types [40]. Alhamoruni et al. [41] demonstrated an enhancing effect of CBD on the mRNA expression of TJPs, including $\mathrm{ZO}-1$, in an in vitro model.

In the present study, CBD and Nano-Se upregulated the expression of ZO-1 and JAM2, which are among the major molecules controlling cell-to-cell adhesion in the gut, while increased expression of TLR4 indicated activation of macrophages as a response to inflammation and augmentation of the host defence against pathogens [42]. Additionally, Nano-Se and Nano-Se + CBD (but not CBD alone) upregulated the expression of the HSP70 gene in the gut tissue. HSP70 belongs to the group of proteins that mediate the stress response in gut tissue, and these proteins are important for adaptation to environmental changes. HSPs are constitutively present in gut tissue and respond to stress-related stimuli [43]. Thus, considering that the HSP70 expression level in our study was similar in both the challenged positive CON group and CBD group but was significantly higher in the remaining treatments, supplementation with Nano-Se was effective in maintaining stress responsiveness in the gut tissue, whereas additional supplementation with CBD did not compromise this response. The action of Se in modulating the response of the broiler gut to challenge has been studied extensively. Se supplementation enhances the production of key components of innate immunity during the initial response to pathogenic stimuli in birds and therefore improves intestinal barrier function [19, 44]. The maximum recommended dose of Se in the broiler diet has been established to be $0.5 \mathrm{mg} / \mathrm{kg}$ of feed [45]. However, recently, we documented that even a dose of $0.7 \mathrm{mg}$ of $\mathrm{Se} / \mathrm{kg}$ of feed was not effective in downregulating oxidative processes in chicken and rat models [46]. The present report demonstrated that Nano-Se, when added at the recommended maximum dose, might have greater potential than other forms of Se to support gut function in chickens. We also provided the first documentation of the potential interaction effect between CBD and Nano-Se in modulating the host response, indicating that the bioactive properties of both agents do not manifest an opposite effect.
Changes in the abundances and metabolic characteristics of the gut microbiota result from their cross-talk with the physiology and diet of the host, which could ultimately influence the specific response of the host to dietary supplements. In contrast to other reports, we did not measure the specific microbiome composition as a response to the dietary treatments, as this parameter varies greatly depending on the conditions [47, 48]. Instead, we investigated changes in the glycolytic activity of the gut microbiota in situ as a mechanism of adaptation for energy uptake to both the applied challenge and dietary treatments, as it is more useful for the present study than changing the bacterial composition. The extracellular enzymatic activity of the microbiome is influenced by the types and abundances of bacterial species residing in the gut and by the rate of release of enzymes from these bacterial cells into the intestinal environment [26]. Therefore, increases in the extracellular activity compared to the total activity (sum of the extracellular and intracellular activities) of those enzymes could be an important adaptive mechanism of intestinal bacteria, as was shown in a different model [49]. We additionally speculate that it could be a potential biomarker of the effect of the applied treatment on the host response. In line with this, the present report demonstrated changes in the activity of selected bacterial enzymes as a result of $C$. perfringens challenge. Particularly indicative was that in 23-day-old birds, there was a shift from intracellular activity towards an increased realizing rate of $\beta$-glucuronidase in the caecal digesta comparing the CON-positive group to the unchallenged $\mathrm{CON}$-negative group. It was reported that the increased activity of $\beta$-glucuronidase may be indicative of increased abundance of Clostridium populations in the gut, and it is associated with the higher risk of glucuronide hydrolysis in the gut lumen, which generates toxic and carcinogenic substances from nontoxic glycosides [50]. In general, the present study demonstrated that the intracellular activity of 5 of the 7 investigated bacterial enzymes was shifted towards an increased rate of release of glycolytic enzymes. More specifically, the greatest shifts were observed in chickens treated with CBD, Nano-Se, and CBD + Nano-Se. Because bacterial enzyme activity depends on the bacterial composition in the digesta, it cannot be excluded that challenging birds with $C$. perfringens altered the microbial balance in the gut; however, the bacterial composition was not analysed in this study. However, a clear response was that treatment with CBD and Nano-Se affected bacterial activity by increasing the enzyme release rate. The increased rate of enzyme release is an adaptive mechanism by which bacteria obtain additional energy from the gut during excessive $C$. perfringens proliferation (the period of challenge). The enzyme release rate could be mediated in 
both a direct manner (through the ability of CBD and Nano-Se to enter bacterial cells) and an indirect manner (through the induced secretion of gut molecules, i.e., mucins, which are metabolized by bacteria) [51]. This hypothesis is supported by the results of other studies that reported the gut microbiome-modulating properties of CBD and Nano-Se [52-55]. Gut microbes metabolize Se for their own needs to synthesize microbial selenoproteins [56]; however, it is not clear how they interact with CBD. Nevertheless, our report demonstrated that CBD induced a shift in the release of bacterial enzymes in the gut, and this action was supported by Nano-Se. Interestingly, our findings indicate that the microbiome did change the enzymatic activity in the post-challenge period because in older birds, the relationship between intra- and extracellular enzymatic activity was markedly changed. An overall shift in extracellular activity but not an increased enzyme release rate was observed in the younger birds. This pattern might be considered beneficial because it indicates that neither CBD nor Nano-Se increases the energy uptake of gut bacteria under optimal conditions (after recovery from challenge), which could be costly regarding energy demands to the host.

In conclusion, this study provided evidence that broilers may be infected with $C$. perfringens with no clinical manifestations, a condition that is potentially hazardous due to possible pathogen transfer to the food chain. Herein, we showed-for the first time in a chicken model-that CBD and Nano-Se may be able to modulate the response of chickens to C. perfringens infection, which may allow time for effective intervention. The beneficial effect of both agents on host physiology was manifested in the support of gut barrier function through increased expression of genes controlling gut integrity. CBD and Nano-Se promoted shifts in bacterial enzyme extracellular activity to increased energy uptake in challenged chickens, and these two agents did not exhibit an opposite effect on mediating the host response to infection. Collectively, these findings indicate that understanding the action mechanisms of CBD and Nano-Se is of great interest for the development of a preventive strategy for NE in broilers.

\section{Abbreviations}

BW: body weight; BWG: body weight gain; CBD: cannabidiol; CON: unchallenged negative control group; FCR: feed conversion ratio; Fint: feed intake; GIT: gastrointestinal tract; GLP2: glucagon-like peptide 2; HSP70: heat shock protein; JAM2: junctional adhesion molecule-2; MMP: matrix metalloproteinase; Nano-Se: selenium nanoparticles; NE: necrotic enteritis; TJPs: tight junction proteins; TLR4: toll-like receptor 4; TFF2: trefoil factor 2; ZO-1: Zonula occludens 1.
}

\section{Acknowledgements}

We would like to acknowledge Janusz Piechota from Amplicon, Poland for the determination of gene expression in this study. We would like to thank
Venessa Eeckhaut from Ghent University for providing the C. perfringens bacteria used in this study.

\section{Authors' contributions}

PK received funding for this study, designed the study concept, performed the chicken trial, and wrote the manuscript. DS and MK performed the chicken trial, collected samples for analysis and participated in the data analysis process. BF performed analyses of collagen concentration and collagenase concentration in the gut samples. JJ performed the analysis of bacterial enzyme activities in caecal digesta. JB acquired and standardized the Cannabis extract. All authors read and approved the final manuscript.

\section{Funding}

This work was supported by the National Science Centre, Grant No. 2018/29/B/NZ9/01351 "Bioactivity of cannabidiol and nano-selenium in the maintenance of gut immunological and integrity potential in chickens".

\section{Availability of data and materials}

The datasets used and/or analysed during the current study are available from the corresponding author on reasonable request.

\section{Ethics approval and consent to participate}

The protocol for this study was approved by the Local Ethical Commission for animal testing at UWM Olsztyn, Poland (Resolution no. 54/2019 from 30 July 2019), and the study was conducted in accordance with the guidelines established by the European Union and Polish Law on Animal Protection.

\section{Consent for publication}

Not applicable.

\section{Competing interests}

The authors declare that they have no competing interests.

\section{Author details}

${ }^{1}$ Department of Animal Nutrition, The Kielanowski Institute of Animal Physiology and Nutrition, Polish Academy of Sciences, Instytucka 3, 05-110 Jabłonna, Poland. ${ }^{2}$ Department of Poultry Science, University of Warmia and Mazury, Oczapowskiego 5, 10-718 Olsztyn, Poland. ${ }^{3}$ Institute of Animal Reproduction and Food Research, Polish Academy of Sciences, Tuwima 10, 10-748 Olsztyn, Poland. ${ }^{4}$ Institute of Natural Fibres and Medicinal Plants, Wojska Polskiego 71b, 60-630 Poznań, Poland.

Received: 25 June 2020 Accepted: 26 October 2020

Published online: 23 November 2020

\section{References}

1. Skinner JT, Bauer S, Young V, Pauling G, Wilson J (2010) An economic analysis of the impact of subclinical (mild) necrotic enteritis in broiler chickens. Avian Dis 54:1237-1240

2. Hussein EOS, Ahmed SH, Abudabos AM, Aljumaah MR, Alkhlulaifi MM, Nassan MA, Suliman GM, Naiel MAE, Swelum AA (2020) Effect of antibiotic, phytobiotic and probiotic supplementation on growth, blood indices and intestine health in broiler chicks challenged with Clostridium perfringens. Animals 10:507

3. Olkowski AA, Wojnarowicz C, Chirino-Trejo M, Drew MD (2006) Responses of broiler chickens orally challenged with Clostridium perfringens isolated from field cases of necrotic enteritis. Res Vet Sci 81:99-108

4. Keyburn AL, Bannam TL, Moore RJ, Rood JI (2010) NetB, a pore-forming toxin from necrotic enteritis strains of Clostridium perfringens. Toxins 2:1913-1927

5. Koutoulis KC, Pappas I, Filioussis G, Athanasiou LV (2015) Pharmacokinetics and clinical assessment of amoxicillin for the control of necrotic enteritis in broiler-breeders under field conditions. Avian Biol Res 8:89-96

6. Keerqin C, Morgan NK, Wu SB, Swick RA, Choct M (2017) Dietary inclusion of arabinoxylo-oligosaccharides in response to broilers challenged with subclinical necrotic enteritis. Br Poult Sci 58:418-424

7. Moore RJ (2016) Necrotic enteritis predisposing factors in broiler chickens. Avian Pathol 45:275-281 
8. Golder HM, Geier MS, Forder REA, Hynd PI, Hughes RJ (2011) Effects of necrotic enteritis challenge on intestinal micro-architecture and mucin profile. Br Poult Sci 52:500-506

9. Forder REA, Nattrass GS, Geier MS, Hughes RJ, Hynd PI (2012) Quantitative analyses of genes associated with mucin synthesis of broiler chickens with induced necrotic enteritis. Poult Sci 91:1335-1341

10. Shojadoost B, Vince AR, Prescott JF (2012) The successful experimental induction of necrotic enteritis in chickens by Clostridium perfringens: a critical review. Vet Res 43:74

11. Immerseel FV, Buck JD, Pasmans F, Huyghebaert G, Haesebrouck F, Ducatelle R (2004) Clostridium perfringens in poultry: an emerging threat for animal and public health. Avian Pathol 33:537-549

12. Kiu R, Brown J, Bedwell H, Leclaire C, Caim S, Pickard D, Dougan G, Dixon RA, Hall $\sqcup$ (2019) Genomic analysis on broiler-associated Clostridium perfringens strains and exploratory caecal microbiome investigation reveals key factors linked to poultry necrotic enteritis. Anim Microbiome 1:12

13. Klein TW, Cabral GA (2006) Cannabinoid-induced immune suppression and modulation of antigen-presenting cells. J Neuroimmune Pharmacol 1:50-64

14. Izzo AA, Sharkey KA (2010) Cannabinoids and the gut: new developments and emerging concepts. Pharmacol Ther 126:21-38

15. Alhamoruni A, Lee AC, Wright KL, Larvin M, O'Sullivan SE (2010) Pharmacological effects of cannabinoids on the Caco-2 cell culture model of intestinal permeability. J Pharmacol Exp Ther 335:92-102

16. Perić L, Milošević N, Žikić D, Kanački Z, Džinić N, Nollet L, Spring P (2009) Effect of selenium sources on performance and meat characteristics of broiler chickens. J Appl Poult Res 18:403-409

17. Yuan D, Zhan XA, Wang YX (2012) Effect of selenium sources on the expression of cellular glutathione peroxidase and cytoplasmic thioredoxin reductase in the liver and kidney of broiler breeders and their offspring. Poult Sci 91:936-942

18. Surai PF, Kochish II, Fisinin VI (2018) Glutathione peroxidases in poultry biology: part 2. Modulation of enzymatic activities. Worlds Poult Sci J 74:239-250

19. Xu S, Lee SH, Lillehoj HS, Hong YH, Bravo D (2015) Effects of dietary selenium on host response to necrotic enteritis in young broilers. Res Vet Sci 98:66-73

20. Wang H, Zhang J, Yu H (2007) Elemental selenium at nano size possesses lower toxicity without compromising the fundamental effect on selenoenzymes: comparison with selenomethionine in mice. Free Radic Biol Med 42:1524-1533

21. Aviagen (2014) Ross 308 broiler nutrition specifications. Aviagen Group, Huntsville

22. Ognik K, Konieczka P, Mikulski D, Jankowski J (2020) The effect of different dietary ratios of lysine and arginine in diets with high or low methionine levels on oxidative and epigenetic DNA damage, the gene expression of tight junction proteins and selected metabolic parameters in Clostridium perfringens-challenged turkeys. Vet Res 51:50

23. Gawronska-Kozak B (2011) Scarless skin wound healing in FOXN1 deficient (nude) mice is associated with distinctive matrix metalloproteinase expression. Matrix Biol 30:290-300

24. Konieczka P, Barszcz M, Kowalczyk P, Szlis M, Jankowski J (2019) The potential of acetylsalicylic acid and vitamin $\mathrm{E}$ in modulating inflammatory cascades in chickens under lipopolysaccharide-induced inflammation. Vet Res 50:65

25. Konieczka P, Mikulski D, Ognik K, Juśkiewicz J, Zduńczyk Z, Józefiak D, Jankowski J (2020) Chemically preserved high-moisture corn in the turkey diet does not compromise performance and maintains the functional status of the gut. Anim Feed Sci Technol 263:114483

26. Gugołek A, Juśkiewicz J, Strychalski J, Zwoliński C, Żary-Sikorska E, Konstantynowicz M (2017) The effects of rapeseed meal and legume seeds as substitutes for soybean meal on productivity and gastrointestinal function in rabbits. Arch Anim Nutr 71:311-326

27. Timbermont L, Haesebrouck F, Ducatelle R, Van Immerseel F (2011) Necrotic enteritis in broilers: an updated review on the pathogenesis. Avian Pathol 40:341-347

28. Gharib-Naseri K, Kheravii SK, Keerqin C, Morgan N, Swick RA, Choct M, Wu SB (2019) Two different Clostridium perfringens strains produce different levels of necrotic enteritis in broiler chickens. Poult Sci 98:6422-6432

29. Calefi AS, da Silva Fonseca JG, Cohn DWH, Honda BTB, Costola-de-Souza C, Tsugiyama LE, Quinteiro-Filho WM, Piantino Ferreira AJ, Palermo-Neto
J (2016) The gut-brain axis interactions during heat stress and avian necrotic enteritis. Poult Sci 95:1005-1014

30. Martin TG, Smyth JA (2010) The ability of disease and non-disease producing strains of Clostridium perfringens from chickens to adhere to extracellular matrix molecules and Caco-2 cells. Anaerobe 16:533-539

31. Wade B, Keyburn AL, Seemann T, Rood Jl, Moore RJ (2015) Binding of Clostridium perfringens to collagen correlates with the ability to cause necrotic enteritis in chickens. Vet Microbiol 180:299-303

32. Van Damme L, Cox N, Callens C, Haesebrouck F, Dargatz M, Ducatelle R, Van Immerseel F, Goossens E (2020) C. perfringens challenge reduces matrix metalloproteinase activity in the jejunal mucosa of Eimeriainfected broiler chickens. Vet Res 51:100

33. Sengupta N, MacDonald TT (2007) The role of matrix metalloproteinases in stromal/epithelial interactions in the gut. Physiology 22:401-409

34. Levin M, Udi Y, Solomonov I, Sagi I (2017) Next generation matrix metalloproteinase inhibitors-novel strategies bring new prospects. Biochim Biophys Acta Mol Cell Res 1864:1927-1939

35. Olkowski AA, Wojnarowicz C, Chirino-Trejo M, Laarveld B, Sawicki G (2008) Sub-clinical necrotic enteritis in broiler chickens: novel etiological consideration based on ultra-structural and molecular changes in the intestinal tissue. Res Vet Sci 85:543-553

36. Aguirre M, Vuorenmaa J, Valkonen E, Kettunen H, Callens C, Haesebrouck F, Ducatelle R, Van Immerseel F, Goossens E (2019) In-feed resin acids reduce matrix metalloproteinase activity in the ileal mucosa of healthy broilers without inducing major effects on the gut microbiota. Vet Res 50:15

37. Kettunen $H$, van Eerden E, Lipiński K, Rinttilä T, Valkonen E, Vuorenmaa $J$ (2017) Dietary resin acid composition as a performance enhancer for broiler chickens. J Appl Anim Nutr 5:e3

38. Awad WA, Hess C, Hess M (2017) Enteric pathogens and their toxininduced disruption of the intestinal barrier through alteration of tight junctions in chickens. Toxins 9:60

39. Tang VW, Goodenough DA (2003) Paracellular ion channel at the tight junction. Biophys J 84:1660-1673

40. De Filippis D, Esposito G, Cirillo C, Cipriano M, De Winter BY, Scuderi C, Sarnelli G, Cuomo R, Steardo L, De Man JG, luvone T (2011) Cannabidiol reduces intestinal inflammation through the control of neuroimmune axis. PLoS One 6:e28159

41. Alhamoruni A, Wright KL, Larvin M, O'Sullivan SE (2012) Cannabinoids mediate opposing effects on inflammation-induced intestinal permeability. Br J Pharmacol 165:2598-2610

42. Chen J, Tellez G, Richards JD, Escobar J (2015) Identification of potential biomarkers for gut barrier failure in broiler chickens. Front Vet Sci 2:14

43. O'Reilly EL, Burchmore RJ, Sparks NH, Eckersall PD (2016) The effect of microbial challenge on the intestinal proteome of broiler chickens. Proteome Sci 15:10

44. Ramasamy KT, Verma P, Reddy MR (2012) Differential gene expression of antimicrobial peptides $\beta$ defensins in the gastrointestinal tract of Salmonella serovar Pullorum infected broiler chickens. Vet Res Commun 36:57-62

45. European Union (2004) List of the Authorized Additives in Feedingstuffs Published in Application of Article 9t (b) of Council Directive 70/524/EEC Concerning Additives in Feeding Stuffs. European Union

46. Konieczka P, Rozbicka-Wieczorek AJ, Czauderna M, Smulikowska S (2017) Beneficial effects of enrichment of chicken meat with n-3 polyunsaturated fatty acids, vitamin $E$ and selenium on health parameters: a study on male rats. Animal 11:1412-1420

47. Stanley D, Geier MS, Hughes RJ, Denman SE, Moore RJ (2013) Highly variable microbiota development in the chicken gastrointestinal tract. PLoS One 8:e84290

48. Yadav S, Jha R (2019) Strategies to modulate the intestinal microbiota and their effects on nutrient utilization, performance, and health of poultry. J Anim Sci Biotechnol 10:2

49. Gugołek A, Juśkiewicz J, Strychalski J, Konstantynowicz M, Zwoliński C (2015) Nutrient digestibility and colonic fermentation processes in species of the families Mustelidae and Canidae fed the same diet. J Exp Zool A Ecol Genet Physiol 323:637-644

50. Beaud D, Tailliez P, Anba-Mondoloni I (2005) Genetic characterization of the $\beta$-glucuronidase enzyme from a human intestinal bacterium, Ruminococcus gnavus. Microbiology 151:2323-2330 
51. Palamidi I, Mountzouris KC (2018) Diet supplementation with an organic acids-based formulation affects gut microbiota and expression of gut barrier genes in broilers. Anim Nutr 4:367-377

52. Cani PD, Plovier H, Van Hul M, Geurts L, Delzenne NM, Druart C, Everard A (2015) Endocannabinoids - at the crossroads between the gut microbiota and host metabolism. Nat Rev Endocrinol 12:133-143

53. Surai PF, Kochish II, Velichko OA (2017) Nano-se assimilation and action in poultry and other monogastric animals: is gut microbiota an answer? Nanoscale Res Lett 12:612

54. Karoly HC, Mueller RL, Bidwell LC, Hutchison KE (2019) Cannabinoids and the microbiota-gut-brain axis: emerging effects of cannabidiol and potential applications to alcohol use disorders. Alcoholism 44:340-353
55. Gangadoo S, Bauer BW, Bajagai YS, Van TTH, Moore RJ, Stanley D (2019) In vitro growth of gut microbiota with selenium nanoparticles. Anim Nutr 5:424-431

56. Peng T, Lin J, Xu YZ, Zhang Y (2016) Comparative genomics reveals new evolutionary and ecological patterns of selenium utilization in bacteria. ISME J 10:2048-2059

\section{Publisher's Note}

Springer Nature remains neutral with regard to jurisdictional claims in published maps and institutional affiliations.
Ready to submit your research? Choose BMC and benefit from:

- fast, convenient online submission

- thorough peer review by experienced researchers in your field

- rapid publication on acceptance

- support for research data, including large and complex data types

- gold Open Access which fosters wider collaboration and increased citations

- maximum visibility for your research: over $100 \mathrm{M}$ website views per year

At BMC, research is always in progress.

Learn more biomedcentral.com/submissions 